\title{
A Biomimetic Strategy for the Synthesis of the
}

\section{Tricyclic Dibenzofuran-1,4-dione Core of}

\section{Popolohuanone E}

\author{
James C. Anderson, * Ross M. Denton and Claire Wilson
}

School of Chemistry, University of Nottingham, Nottingham, NG7 2RD, UK

j.anderson@nottingham.ac.uk

Index

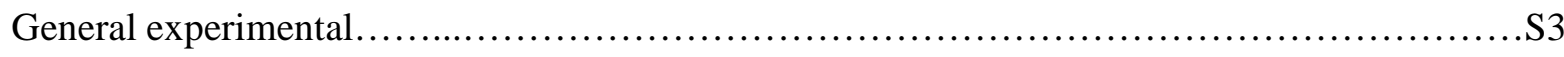

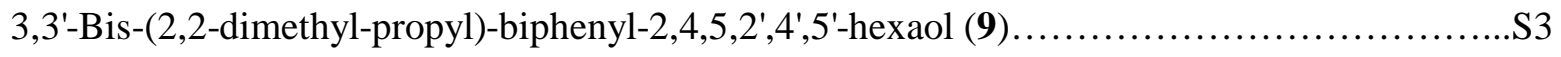

3,3'-Bis-(2,2-dimethyl-propyl)-4,4'-dihydroxy-bicyclohexyl-3,6,3',6'-tetraene-2,5,2',5'-tetraone

(10). .S3

2,6-Bis-(2,2-dimethyl-propyl)-3,7,8-trihydroxy-dibenzofuran-1,4-dione (11)..................S3

2,6-Bis-(2,2-dimethyl-propyl)-3,7,8-trimethoxy-dibenzofuran-1,4-dione (12).................S3

3,7,8-Tris-allyloxy-2,6-bis-(2,2-dimethyl-propyl)-dibenzofuran-1,4-dione (13) ...............S4

3,7,8-Tris-benzyloxy-2,6-bis-(2,2-dimethyl-propyl)-dibenzofuran-1,4-dione (14)..............S4

2,6-Bis-(2,2-dimethyl-propyl)-7,8-dihydroxy-3-methoxy-dibenzofuran-1,4-dione (15)...........S4

2,6-Bis-(2,2-dimethyl-propyl)-3,7,8-trihydroxy-dibenzofuran-1,4-dione (11) from (15)..........S4

2,6-Bis-(2,2-dimethyl-propyl)-3,7,8-trihydroxy-dibenzofuran-1,4-dione (11) from (14).........S4

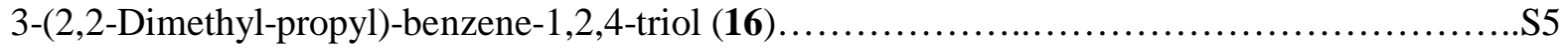

3,3'-Bis-(2,2-dimethyl-propyl)-4,4'-dihydroxy-bicyclohexyl-3,6,3',6'-tetraene-2,5,2',5'-

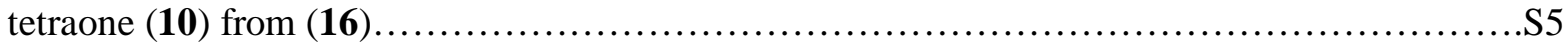

Copies of $1 \mathrm{H}$ and 13C NMR spectra:

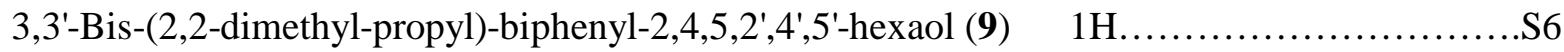

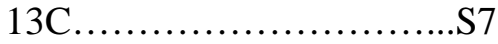

3,3'-Bis-(2,2-dimethyl-propyl)-4,4'-dihydroxy-bicyclohexyl-3,6,3',6'-tetraene-2,5,2',5'-tetraone

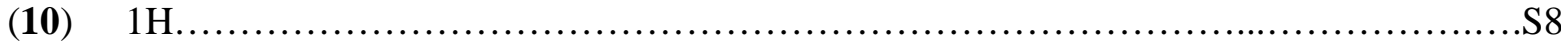

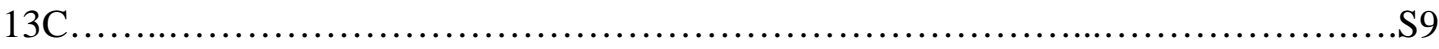


2,6-Bis-(2,2-dimethyl-propyl)-3,7,8-trihydroxy-dibenzofuran-1,4-dione (11) $\quad 1 \mathrm{H} \ldots \ldots \ldots . . . \mathrm{S} 10$ $13 \mathrm{C} \ldots \ldots . . . . . \mathrm{S} 11$

2,6-Bis-(2,2-dimethyl-propyl)-3,7,8-trimethoxy-dibenzofuran-1,4-dione (12) $\quad 1 \mathrm{H} \ldots \ldots . . . . . \mathrm{S} 12$ $13 \mathrm{C} \ldots \ldots . . . . \mathrm{S} 13$

3,7,8-Tris-allyloxy-2,6-bis-(2,2-dimethyl-propyl)-dibenzofuran-1,4-dione (13) $\quad 1 \mathrm{H} \ldots \ldots \ldots . . . \mathrm{S} 14$ $13 \mathrm{C} \ldots \ldots \ldots . . . . \mathrm{S} 15$

3,7,8-Tris-benzyloxy-2,6-bis-(2,2-dimethyl-propyl)-dibenzofuran-1,4-dione (14) $1 \mathrm{H} \ldots \ldots \ldots . . . \mathrm{S} 16$ $13 \mathrm{C} \ldots \ldots \ldots . . . . \mathrm{S} 17$

2,6-Bis-(2,2-dimethyl-propyl)-7,8-dihydroxy-3-methoxy-dibenzofuran-1,4-dione (15) 1H....S18

$13 \mathrm{C}$...S19

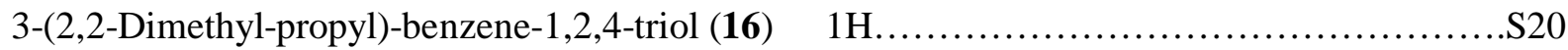

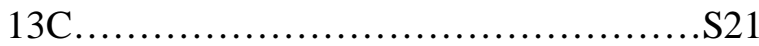




\section{General experimental.}

For all non-aqueous chemistry, glassware was rigorously flame-dried and an inert $\left(\mathrm{N}_{2}\right)$ atmosphere was maintained throughout. Thin layer chromatography was carried out on silica-aluminium plates and were visualised using ultra-violet light $(254 \mathrm{~nm})$ and/or anisaldehyde or $\mathrm{KMnO}_{4}$ solutions, as appropriate. For flash column chromatography silica gel was used and direct column loading was employed in all cases. NMR data was collected at 400 or $500 \mathrm{MHz}$. Data was manipulated directly from the spectrometer or via a networked PC with appropriate software. All samples were analysed in $\mathrm{CDCl}_{3}$ unless otherwise stated. Reference values for residual solvent was taken as $\delta=7.27$ $\left(\mathrm{CDCl}_{3}\right)$ for ${ }^{1} \mathrm{H}-\mathrm{NMR} ; \delta=77.16\left(\mathrm{CDCl}_{3}\right)$ for ${ }^{13} \mathrm{C}-\mathrm{NMR}$. Multiplicities for coupled signals are denoted thus: $\mathrm{s}=$ singlet, $\mathrm{d}=$ doublet, $\mathrm{t}=$ triplet, $\mathrm{q}=$ quartet, quin=quintet, $\mathrm{sex}=$ sextet, br=broad signal, ap=apparent and are given in $\mathrm{Hz} .{ }^{13} \mathrm{C}$ multiplicities were assigned using a DEPT sequence. Where appropriate, COSY, HMQC, HMBC and NOE experiments were performed to aid assignment. High-resolution mass spectrometric data are quoted to four decimal places $(0.1 \mathrm{mDa})$ with error limits for acceptance of $+/-5.0 \mathrm{ppm}$ (defined as calcd./found mass $10^{-6}$ ). Infrared data was collected using sodium chloride discs. Both oils and solids were analysed neat. All solvents and reagents were used as supplied or purified using standard techniques.

3,3'-Bis-(2,2-dimethyl-propyl)-biphenyl-2,4,5,2',4',5'-hexaol (9). To a solution of $8^{1}$ ( $2.50 \mathrm{~g}$, $5.47 \mathrm{mmol})$ in $\mathrm{CH}_{2} \mathrm{Cl}_{2}(12.5 \mathrm{~mL})$ was added TMSI $(25.0 \mathrm{~mL}, 106 \mathrm{mmol})$ and the resultant solution was heated at reflux and stirred for $15 \mathrm{~h}$. After this time $\mathrm{MeOH}(25 \mathrm{~mL})$ was added cautiously and the reaction mixture concentrated in vacuo. The residue was dissolved in $\mathrm{Et}_{2} \mathrm{O}$ and washed with satd. aq. $\mathrm{Na}_{2} \mathrm{SO}_{3}$, brine $(25 \mathrm{~mL})$, dried $\left(\mathrm{MgSO}_{4}\right)$, filtered and the solvent removed in vacuo giving a white solid $(2.04 \mathrm{~g}, 96 \%)$ which was used without further purification. Mp $71{ }^{\circ} \mathrm{C} \mathrm{dec}$; IR $v_{\max } 3371,2949$, 1614, 1475, 1456, 1294, 1230, $965 \mathrm{~cm}^{-1} ;{ }^{1} \mathrm{H}$ NMR $\delta 0.99(18 \mathrm{H}, \mathrm{s}), 2.72(4 \mathrm{H}, \mathrm{s}), 6.58(2 \mathrm{H}, \mathrm{s}), 7.01$ $(2 \mathrm{H}, \mathrm{s}), 7.98(2 \mathrm{H}, \mathrm{s}) ;{ }^{13} \mathrm{C} \mathrm{NMR} \delta 30.1,34.0,36.8,114.9,116.1,117.5,138.6,144.9,146.2 ; \mathrm{m} / z\left(\mathrm{EI}^{+}\right)$ $390\left(\mathrm{M}^{+}, 64 \%\right), 333$ (40\%), 276 (9\%), 57 (100\%); HRMS $\mathrm{C}_{22} \mathrm{H}_{30} \mathrm{O}_{6}$ calcd. 390.20425, found 390.20380 .

\section{3,3'-Bis-(2,2-dimethyl-propyl)-4,4'-dihydroxy-bicyclohexyl-3,6,3',6'-tetraene-2,5,2',5'-}

tetraone (10). To a suspension of $9(2.13 \mathrm{~g}, 5.23 \mathrm{mmol})$ in $\mathrm{CH}_{2} \mathrm{Cl}_{2}(400 \mathrm{~mL})$ was added $\mathrm{FeCl}_{3}(67.8$ g, $20.9 \mathrm{mmol}, 5 \% \mathrm{wt}$ on $\mathrm{SiO}_{2}$ ) and the reaction then stirred for $5 \mathrm{~min}$. The reaction mixture was then filtered and concentrated in vacuo. The residue was dissolved in EtOAc:petroleum ether (3:7) and decolourising charcoal $(50 \mathrm{mg})$ was added, the mixture filtered and concentrated in vacuo. Recrystallisation (petroleum ether) of the residue gave $10(1.72 \mathrm{~g}, 85 \%)$ as yellow needles mp 191 ${ }^{\circ} \mathrm{C}$ dec; IR $v$ max (neat) 3420, 2963,1655, 1380, $1059 \mathrm{~cm}^{-1} ;{ }^{1} \mathrm{H}$ NMR $\delta 0.94(18 \mathrm{H}, \mathrm{s}), 2.41(4 \mathrm{H}, \mathrm{s})$, $6.71(2 \mathrm{H}, \mathrm{s}), 7.01(2 \mathrm{H}, \mathrm{s}) ;{ }^{13} \mathrm{C}$ NMR $\delta 30.2,33.9,35.9,121.1,130.6,144.1,152.3,182.2,185.5 ; \mathrm{m} / \mathrm{z}$ $\left(\mathrm{EI}^{+}\right) 386\left(\mathrm{M}^{+}, 10 \%\right), 330(57 \%)$; HRMS $\mathrm{C}_{22} \mathrm{H}_{26} \mathrm{O}_{6}$ calcd. 386.17294, found 386.17244.

2,6-Bis-(2,2-dimethyl-propyl)-3,7,8-trihydroxy-dibenzofuran-1,4-dione (11). To a solution of $10(100 \mathrm{mg}, 0.26 \mathrm{mmol})$ in acetone $(10 \mathrm{~mL})$ was added $\mathrm{K}_{2} \mathrm{CO}_{3}(107 \mathrm{mg}, 0.78 \mathrm{mmol})$ and the resulting mixture was stirred for $15 \mathrm{~h}$. The reaction was then diluted with $\mathrm{Et}_{2} \mathrm{O}$ and washed with $1 \mathrm{M}$ $\mathrm{HCl}$, dried $\left(\mathrm{MgSO}_{4}\right)$ and the solvent removed in vacuo. Purification by recrystalisation (EtOAc:pet. ether) gave 11 (79 mg, 79\%) as dark purple needles Mp >250 ${ }^{\circ} \mathrm{C}$; IR $v$ max $3521,3253,2957,1661$, 1623, 1608, 1563, 1458, 1327, 1279, 1216, $1073 \mathrm{~cm}^{-1} ;{ }^{1} \mathrm{H}$ NMR $\delta 0.92(9 \mathrm{H}, \mathrm{s}), 0.97(9 \mathrm{H}, \mathrm{s}), 2.36$ $(2 \mathrm{H}, \mathrm{s}), 2.73(2 \mathrm{H}, \mathrm{s}), 7.30(1 \mathrm{H}, \mathrm{s}), 9.28(1 \mathrm{H}, \mathrm{s}), 10.26(1 \mathrm{H}, \mathrm{s}), 10.75(1 \mathrm{H}, \mathrm{s}) ;{ }^{13} \mathrm{C}$ NMR $\delta 30.6,31.1$, 34.2, 34.3, 35.8, 37.5, 103.7, 111.5, 114.3, 118.6, 124.0, 146.7, 148.5, 149.0, 153.3, 156.2, 171.9, 185.5; $\mathrm{m} / z\left(\mathrm{CI}^{+}\right) 386\left(100 \%, \mathrm{M}^{+}\right)$; HRMS C ${ }_{22} \mathrm{H}_{26} \mathrm{O}_{6}$ calcd. 386.17294, found 386.17249; Anal. calcd. for $\mathrm{C}_{22} \mathrm{H}_{26} \mathrm{O}_{6}$ : $\mathrm{C} 68.38 ; \mathrm{H}, 6.78$ found $\mathrm{C} 68.52 ; \mathrm{H} 6.60 \%$.

2,6-Bis-(2,2-dimethyl-propyl)-3,7,8-trimethoxy-dibenzofuran-1,4-dione (12). To a solution of biquinone $10(350 \mathrm{mg}, 0.90 \mathrm{mmol})$ in acetone $(36 \mathrm{~mL})$ was added $\mathrm{K}_{2} \mathrm{CO}_{3}(373 \mathrm{mg}, 2.70 \mathrm{mmol})$, MeI $(0.30 \mathrm{~mL}, 4.50 \mathrm{mmol})$ and the resulting mixture was stirred for $36 \mathrm{~h}$ at $\mathrm{rt}$. The reaction was then diluted with $\mathrm{Et}_{2} \mathrm{O}$ and washed with brine. The organic layer was then dried $\left(\mathrm{MgSO}_{4}\right)$ and the solvent 
removed in vacuo. Purification by flash column chromatography (silica, 30\% EtOAc in pet. ether) followed by recrystallisation $\left(\mathrm{CH}_{3} \mathrm{OH}\right)$ afforded $12(285 \mathrm{mg}, 74 \%)$ as dark red needles $\mathrm{mp} 143$ $144^{\circ} \mathrm{C}$; IR $v_{\max } 2963,1670,1651,1564,1458,1261,1272,1073,1026 \mathrm{~cm}^{-1} ;{ }^{1} \mathrm{H}$ NMR $\delta 1.02(9 \mathrm{H}$, s), $1.08(9 \mathrm{H}, \mathrm{s}), 2.57(2 \mathrm{H}, \mathrm{s}), 2.94(2 \mathrm{H}, \mathrm{s}), 3.93(3 \mathrm{H}, \mathrm{s}), 4.04(3 \mathrm{H}, \mathrm{s}), 4.19(3 \mathrm{H}, \mathrm{s}), 7.52(1 \mathrm{H}, \mathrm{s}) ;{ }^{13} \mathrm{C}$ NMR $\delta 30.3,30.8,33.7,34.0,36.1,37.8,56.5,61.3,61.5,101.5,117.6,119.5,122.7,131.0,150.2$, 150.4, 152.2, 152.9, 157.0, 172.9, 184.5; m/z (EI') $428\left(\mathrm{M}^{+}, 74 \%\right), 372(100 \%) ; \mathrm{HRMS} \mathrm{C}_{25} \mathrm{H}_{32} \mathrm{O}_{6}$

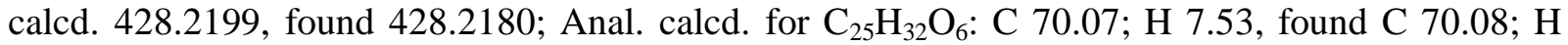
$7.49 \%$.

3,7,8-Tris-allyloxy-2,6-bis-(2,2-dimethyl-propyl)-dibenzofuran-1,4-dione (13). Prepared from $10(350 \mathrm{mg}, 0.91 \mathrm{mmol})$ by an identical method to the preparation of 12, but using allyl bromide $(1.54 \mathrm{~mL}, 18.2 \mathrm{mmol})$ and $n$-Bu $\mathrm{Bu}_{4} \mathrm{NI}(15.0 \mathrm{mg}, 45.0 \mu \mathrm{mol})$ to give $13(355 \mathrm{mg}, 77 \%)$ as dark red needles mp $161{ }^{\circ} \mathrm{C}$; IR $v_{\max } 2952,1670,1657,1561,1422,1275,1219,1068,924 \mathrm{~cm}^{-1}$; ${ }^{1} \mathrm{H}$ NMR $\delta$ $0.96(9 \mathrm{H}, \mathrm{s}), 0.99(9 \mathrm{H}, \mathrm{s}), 2.53(2 \mathrm{H}, \mathrm{s}), 2.88(2 \mathrm{H}, \mathrm{s}), 4.56(2 \mathrm{H}, \mathrm{d}, J 6.0 \mathrm{~Hz}), 4.67(2 \mathrm{H}, \mathrm{d}, J 5.0 \mathrm{~Hz})$, $4.85(2 \mathrm{H}, J 6.0 \mathrm{~Hz}), 5.28-5.50(6 \mathrm{H}, \mathrm{m}), 6.08-6.14(3, \mathrm{~m}), 7.46(1 \mathrm{H},) ;{ }^{13} \mathrm{C}$ NMR $\delta 30.4,30.6,33.6$, $33.8,36.1,37.8,70.0,74.1,74.2,102.9,117.6,117.7,118.1,118.7,120.1,122.7,132.0,132.8$, 133.5, 134.1, 149.4, 150.2, 151.8, 152.2, 159.2, 172.9, 184.4; HRMS $\left(\mathrm{CI}^{+}\right) \mathrm{C}_{31} \mathrm{H}_{39} \mathrm{O}_{6}$ calcd. 507.2747, found 507.2748 $\left(\mathrm{M}^{+}\right)$; Anal. calcd. for $\mathrm{C}_{31} \mathrm{H}_{39} \mathrm{O}_{6}: \mathrm{C} 73.49 ; \mathrm{H} \mathrm{7.56}$, found $\mathrm{C} 73.15 ; \mathrm{H}$ $7.46 \%$.

3,7,8-Tris-benzyloxy-2,6-bis-(2,2-dimethyl-propyl)-dibenzofuran-1,4-dione (14). Prepared from $10(310 \mathrm{mg}, 0.803 \mathrm{mmol})$ by an identical method to the preparation of 12, but using benzyl bromide $(1.90 \mathrm{~mL}, 16.0 \mathrm{mmol})$ and TBAI $(29.5 \mathrm{mg}, 80.0 \mu \mathrm{mol})$. Purification by flash column chromatography (silica, 30\% EtOAc/pet. ether) gave $14(159 \mathrm{mg}, 69 \%)$. as red needles mp $98{ }^{\circ} \mathrm{C}$; IR $v_{\max } 2956,1672,1654,1564,1276,1219,1068,1025,986,732 \mathrm{~cm}^{-1} ;{ }^{1} \mathrm{H}$ NMR $\delta 0.95(9 \mathrm{H}, \mathrm{s}), 0.98$ $(9 \mathrm{H}, \mathrm{s}), 2.47(2 \mathrm{H}, \mathrm{s}), 2.81(2 \mathrm{H}, \mathrm{s}), 5.05(2 \mathrm{H}, \mathrm{s}), 5.23(2 \mathrm{H}, \mathrm{s}), 5.38(2 \mathrm{H}, \mathrm{s}), 7.34-7.51(15 \mathrm{H}, \mathrm{m}), 7.63$ $(1 \mathrm{H}, \mathrm{s}) ;{ }^{13} \mathrm{C}$ NMR $\delta 30.1,30.6,33.7,33.9,36.0,37.8,103.0,117.1,120.4,122.8,127.9,128.1$, $128.2,128.3,128.4,128.5,128.6,128.7,132.4,136.4,136.8,137.4,149.6,150.3,152.0,152.3$, 156.2; m/z $\left(\mathrm{CI}^{+}\right) 656\left(\mathrm{M}^{+}, 8 \%\right), 565$ (10\%), 509 (5\%), $475(5 \%), 91(100 \%) ; \mathrm{HRMS} \mathrm{C}_{43} \mathrm{H}_{44} \mathrm{O}_{6}$ cacld. 656.31379, found 656.31519.

2,6-Bis-(2,2-dimethyl-propyl)-7,8-dihydroxy-3-methoxy-dibenzofuran-1,4-dione (15). To a solution of $13(73.0 \mathrm{mg}, 0.14 \mathrm{mmol})$ in $\mathrm{MeOH}(2 \mathrm{~mL})$ was added $\mathrm{K}_{2} \mathrm{CO}_{3}(173 \mathrm{mg}, 1.26 \mathrm{mmol})$ and the reaction stirred at rt. After $5 \mathrm{~min} \mathrm{Pd}\left(\mathrm{PPh}_{3}\right)_{4}(1.60 \mathrm{mg}, 14.0 \mu \mathrm{mol})$ was added and the reaction was stirred for a further $1 \mathrm{~h}$ at $\mathrm{rt}$. The reaction was then diluted with $\mathrm{Et}_{2} \mathrm{O}$, washed with $1 \mathrm{M} \mathrm{HCl}$, brine, dried $\left(\mathrm{MgSO}_{4}\right)$ and the solvent removed in vacuo. Purification by flash column chromatography (silica, 30\% EtOAc in pet. ether) gave $15(50 \mathrm{mg}, 88 \%)$ as a dark purple solid $\mathrm{mp}$ $>250{ }^{\circ} \mathrm{C}$; IR $v_{\max }\left(\mathrm{CHCl}_{3}\right) 3524,3294,1641,1561,1456,1366,1077,989 \mathrm{~cm}^{-1} ;{ }^{1} \mathrm{H}$ NMR $\delta 0.98$ $(9 \mathrm{H}, \mathrm{s}), 1.05(9 \mathrm{H}, \mathrm{s}), 2.51(2 \mathrm{H}, \mathrm{s}), 2.92(2 \mathrm{H}, \mathrm{s}), 5.59(1 \mathrm{H}, \mathrm{s}), 6.11(1 \mathrm{H}, \mathrm{s}), 7.46(1 \mathrm{H}, \mathrm{s}) ;{ }^{13} \mathrm{C} \mathrm{NMR}$ (acetone- $\left.d_{6}\right) \delta 30.1,30.4,33.4,34.0,35.9,37.3,61.1,103.9,111.6,114.2,123.1,130.3,145.5$, 148.1, 157.9, 172.6, 184.9; m/z $\left(\mathrm{CI}^{+}\right) 400\left(\mathrm{M}^{+}, 100 \%\right), 385$ (22\%); HRMS $\mathrm{C}_{23} \mathrm{H}_{28} \mathrm{O}_{6}$ calcd. 400.18859 , found 400.18870 .

2,6-Bis-(2,2-dimethyl-propyl)-3,7,8-trihydroxy-dibenzofuran-1,4-dione (11) from (15). To a solution of $15(50.0 \mathrm{mg}, 0.13 \mathrm{mmol})$ in DMF (5 mL) was added ${ }^{\mathrm{n}} \mathrm{BuSNa}(146 \mathrm{mg}, 1.30 \mathrm{mmol})$ and the reaction heated to $90^{\circ} \mathrm{C}$ and stirred for $16 \mathrm{~h}$. After this time the reaction mixture was diluted with EtOAc, washed with water, then brine, dried $\left(\mathrm{MgSO}_{4}\right)$, filtered and the solvent removed in vacuo. Purification by flash column chromatography (EtOAc:pet. ether:AcOH 50:50:0.5) gave 11 (43 $\mathrm{mg}, 89 \%)$ as a dark purple solid with identical spectral data to that prepared earlier. 
2,6-Bis-(2,2-dimethyl-propyl)-3,7,8-trihydroxy-dibenzofuran-1,4-dione (11) from (14). To a solution of $14(55.0 \mathrm{mg}, 83.8 \mu \mathrm{mol})$ in EtOH $(2 \mathrm{~mL})$ was added $\mathrm{HCO}_{2} \mathrm{NH}_{4}(52.8 \mathrm{mg}, 838 \mu \mathrm{mol})$ followed by $10 \% \mathrm{Pd}-\mathrm{C}(2.50 \mathrm{mg})$ and the reaction stirred for $20 \mathrm{~min}$. The reaction mixture was then filtered and the solvent removed in vacuo. Purification by flash column chromatography (EtOAc:pet. ether:AcOH 50:50:0.5) gave $11(31 \mathrm{mg}, 95 \%)$ as a dark purple solid with identical spectral data to that prepared earlier.

3-(2,2-Dimethyl-propyl)-benzene-1,2,4-triol (16). To a solution of $\mathbf{7}^{1}(240 \mathrm{mg}, 1.01 \mathrm{mmol})$ in $\mathrm{CH}_{2} \mathrm{Cl}_{2}$ was added TMSI $(2.00 \mathrm{~mL}, 14.0 \mathrm{mmol})$ and the reaction heated at reflux with stirring. After $15 \mathrm{~h} \mathrm{MeOH}(3 \mathrm{~mL})$ was added cautiously and the reaction mixture concentrated in vacuo. The residue was dissolved in $\mathrm{Et}_{2} \mathrm{O}$, washed with satd. aq. $\mathrm{Na}_{2} \mathrm{SO}_{3}$, brine, dried $\left(\mathrm{MgSO}_{4}\right)$ filtered and the solvent removed in vacuo affording $16(193 \mathrm{mg}, 98 \%)$ as a white solid which was used without further purification. $\mathrm{Mp} 89{ }^{\circ} \mathrm{C}$; IR $v_{\max } 3501,3344,2950,1492,1460,1258,1217,1156,1049,957$ $\mathrm{cm}^{-1} ;{ }^{1} \mathrm{H}$ NMR $\delta 1.01(9 \mathrm{H}, \mathrm{s}), 2.59(2 \mathrm{H}, \mathrm{s}), 4.44(1 \mathrm{H}, \mathrm{s}), 4.71(1 \mathrm{H}, \mathrm{s}), 5.34(1 \mathrm{H}, \mathrm{s}), 6.27(1 \mathrm{H}, \mathrm{d} J$ 9.0), $6.63(1 \mathrm{H}, \mathrm{d} J 9.0) ;{ }^{13} \mathrm{C}$ NMR $\delta 31.5,35.6,37.9,106.9,114.3,116.8,139.5,147.2,51.8 ; \mathrm{m} / \mathrm{z}$ $\left(\mathrm{EI}^{+}\right) 196\left(\mathrm{M}^{+}, 30 \%,\right), 139$ (64\%); HRMS C${ }_{11} \mathrm{H}_{16} \mathrm{O}_{3}$ calcd. 196.10994, found 196.11005.

\section{3,3'-Bis-(2,2-dimethyl-propyl)-4,4'-dihydroxy-bicyclohexyl-3,6,3',6'-tetraene-2,5,2',5'-} tetraone (10) from (16). A degassed solution of $16(150 \mathrm{mg}, 0.77 \mathrm{mmol})$ in $\mathrm{CH}_{2} \mathrm{Cl}_{2}: \mathrm{CH}_{3} \mathrm{CN}(3 \mathrm{~mL}$ : $1 \mathrm{~mL})$ was added via cannula to a suspension of $\mathrm{FeCl}_{3}\left(9.98 \mathrm{~g}, 3.08 \mathrm{mmol}\right.$ of a $5 \%$ wt. on $\left.\mathrm{SiO}_{2}\right)$ in $\mathrm{CH}_{2} \mathrm{Cl}_{2}(40 \mathrm{~mL})$ and the reaction mixture was stirred for $5 \mathrm{~min}$. The reaction mixture was then filtered and concentrated in vacuo. The residue was dissolved in EtOAc:petroleum ether (30 mL: 70 $\mathrm{mL})$ and decolourising charcoal $(10 \mathrm{mg}$ ) was added. The mixture was filtered and the solvents removed in vacuo. Recrystallisation (EtOAc:pet. ether) of the residue gave $\mathbf{1 0}$ as yellow needles (122 mg, 81\%) with identical spectral data to that prepared earlier.

\footnotetext{
${ }^{1}$ Anderson, J.C.; Pearson, D.J. J. Chem. Soc., Perkin Trans. 11998, 2023
} 

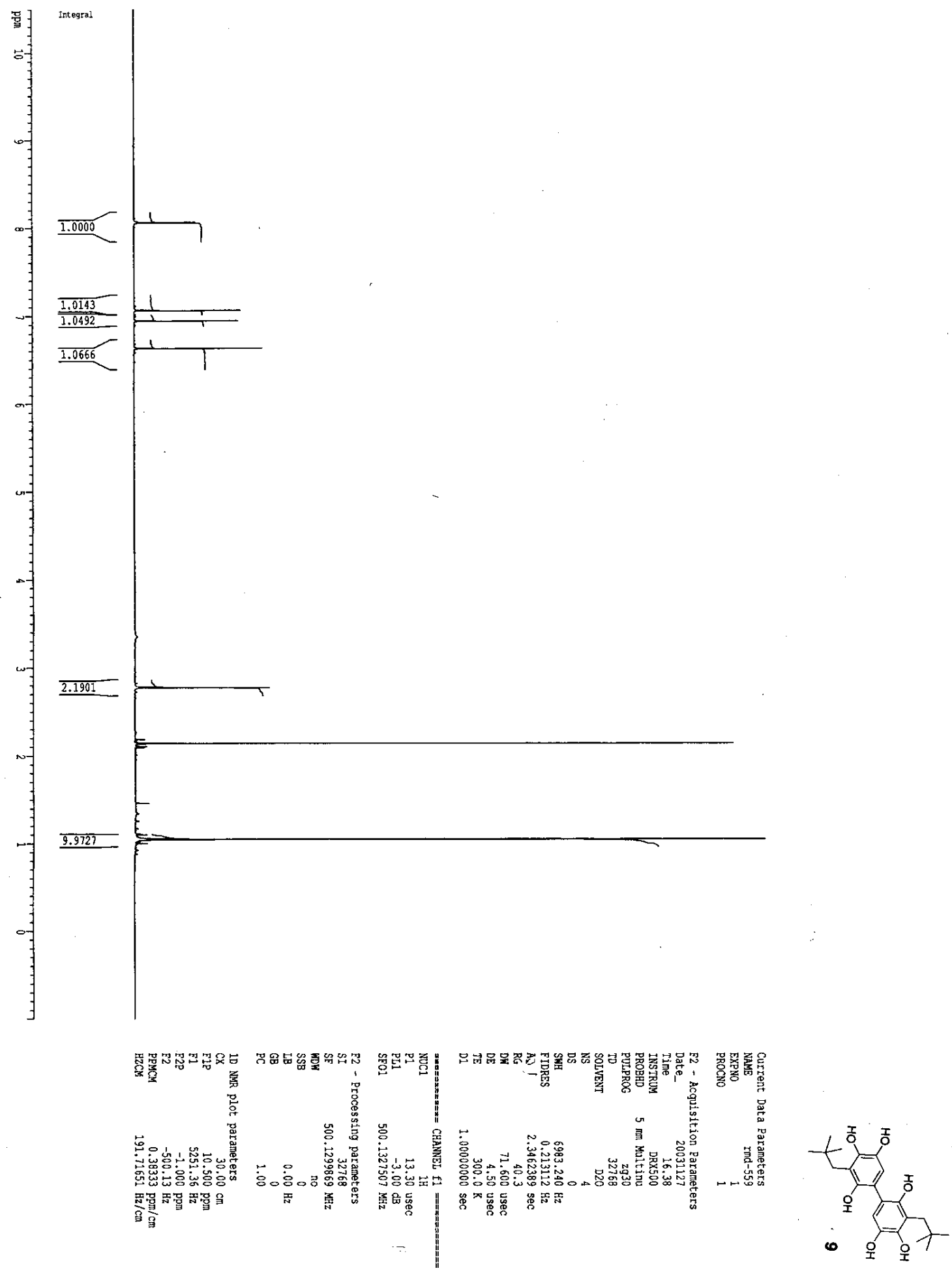


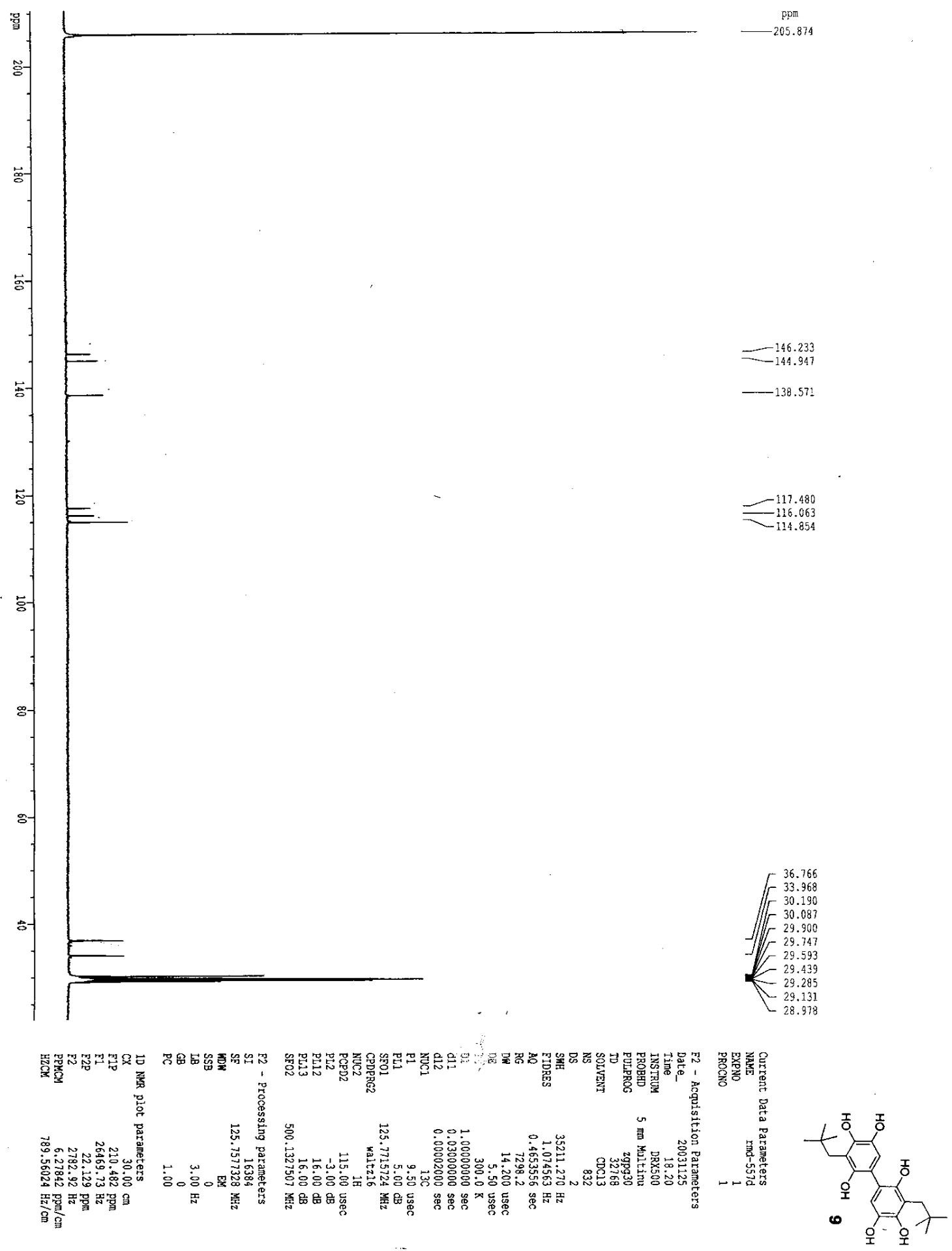



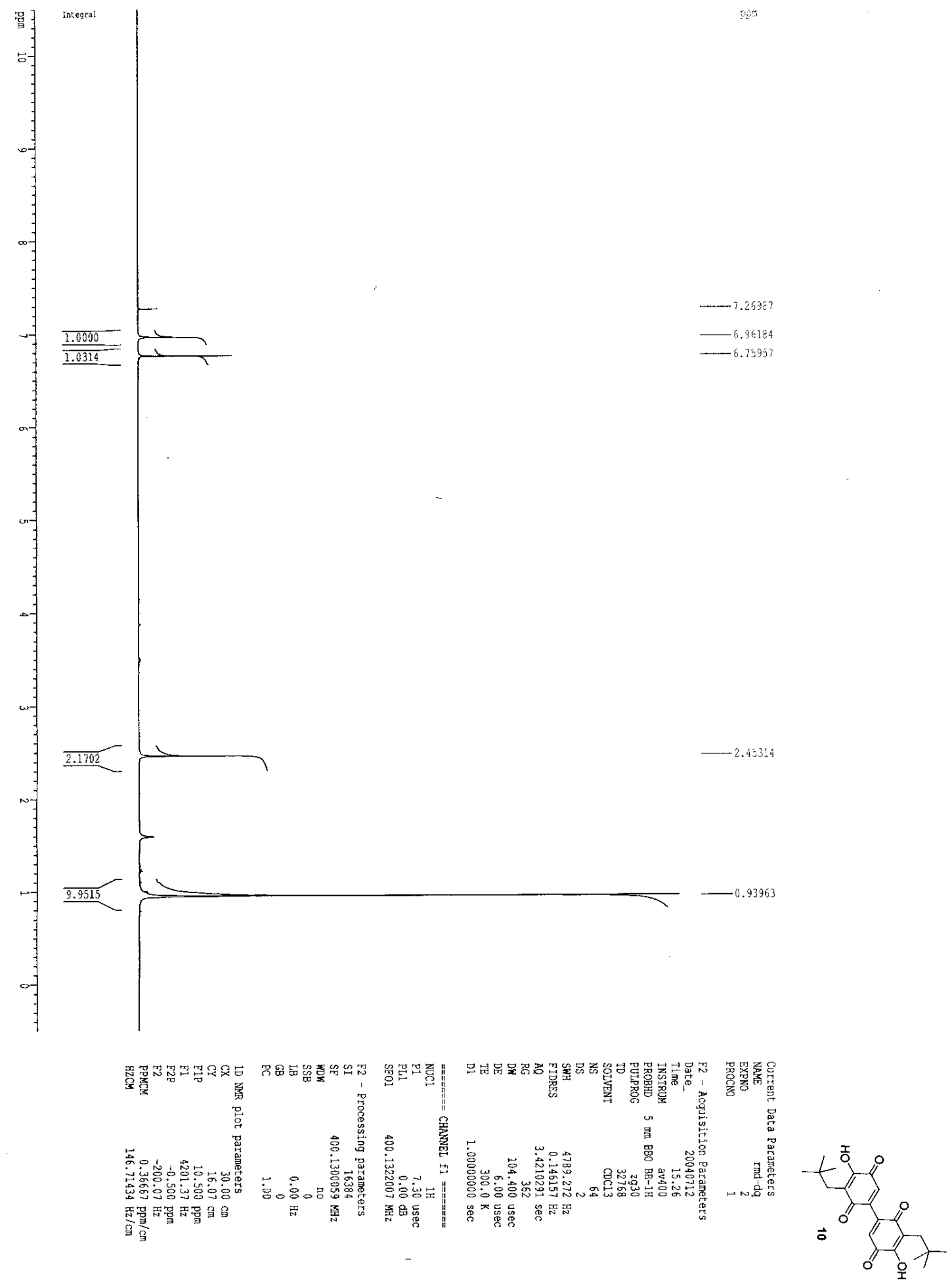


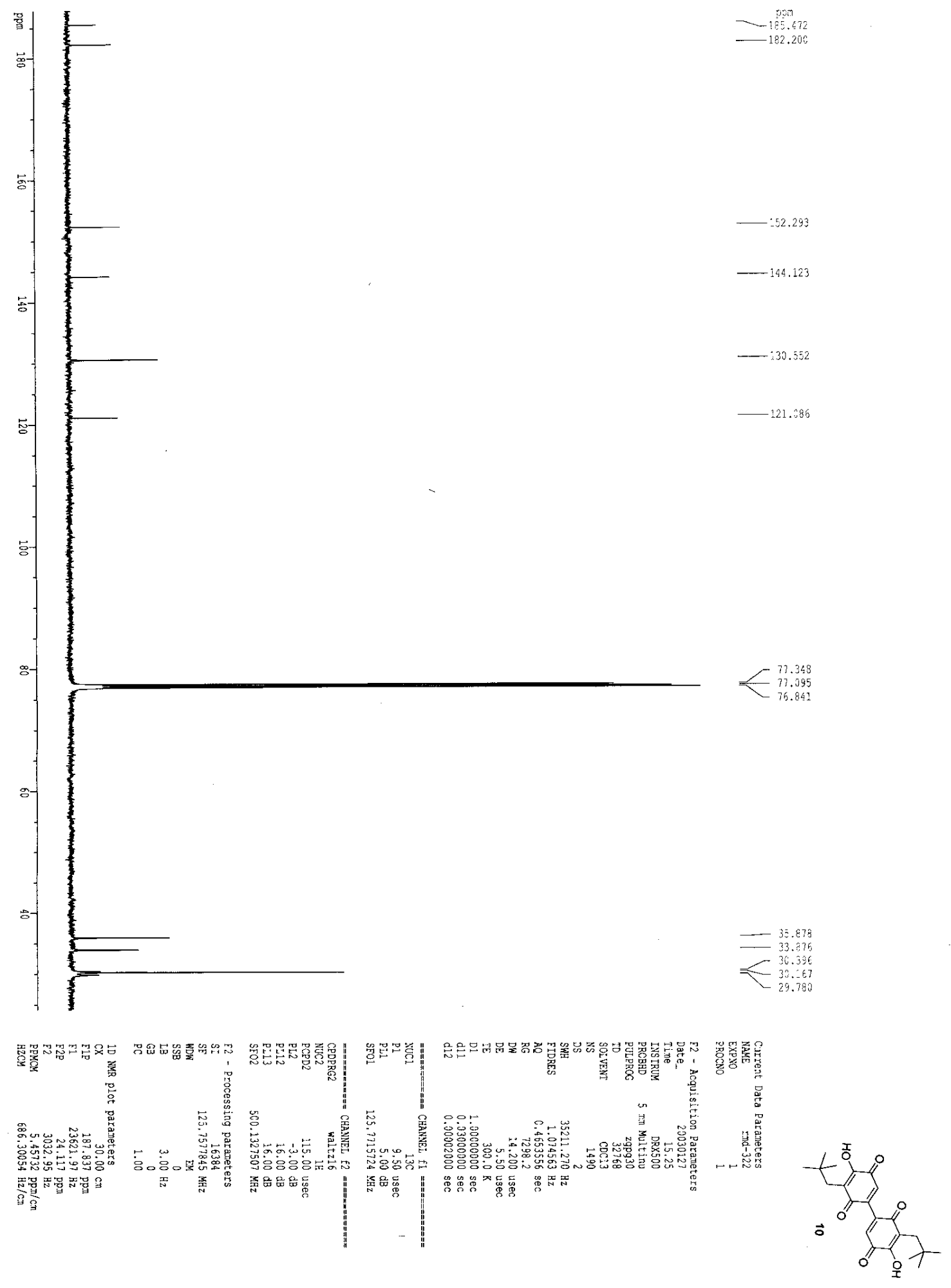



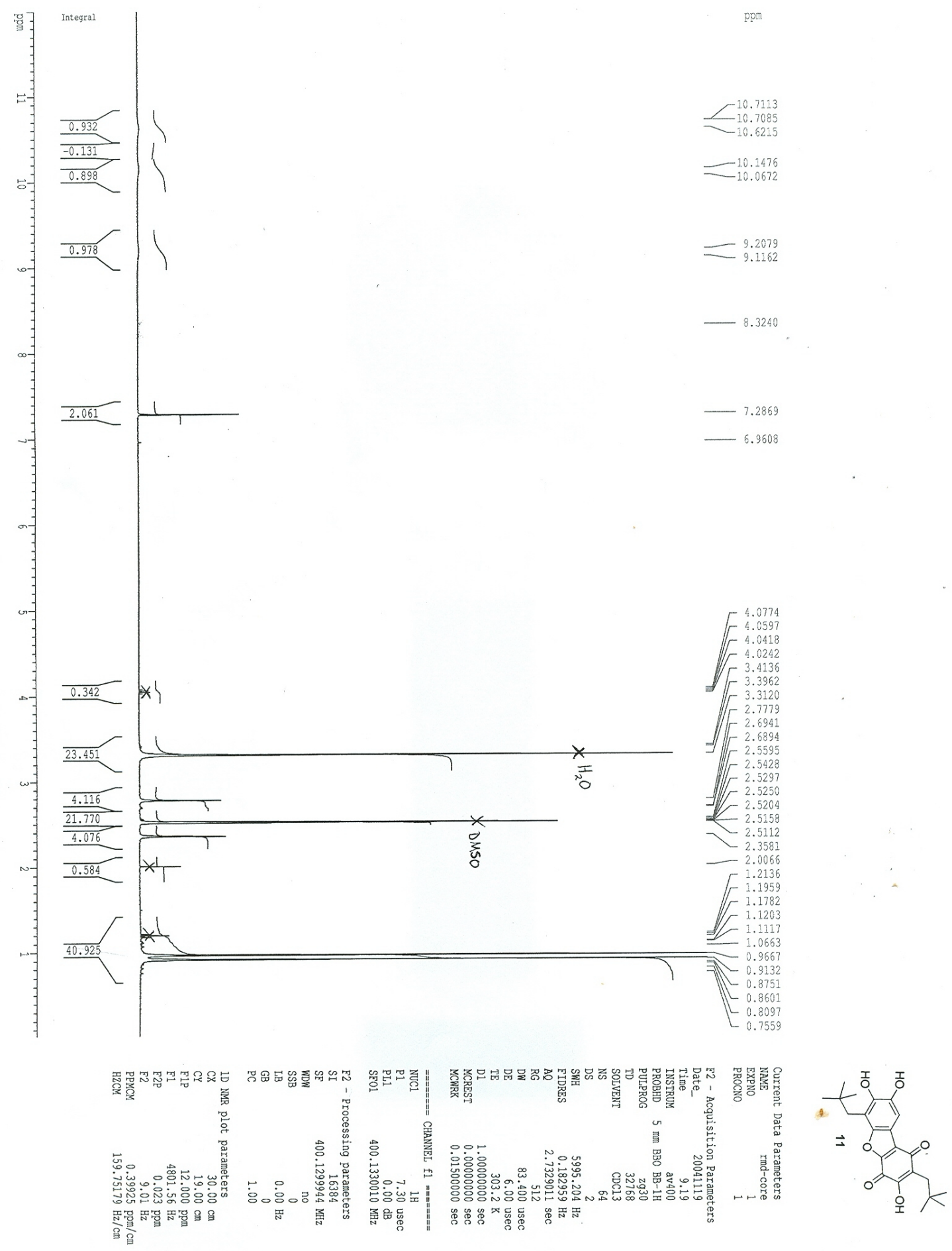


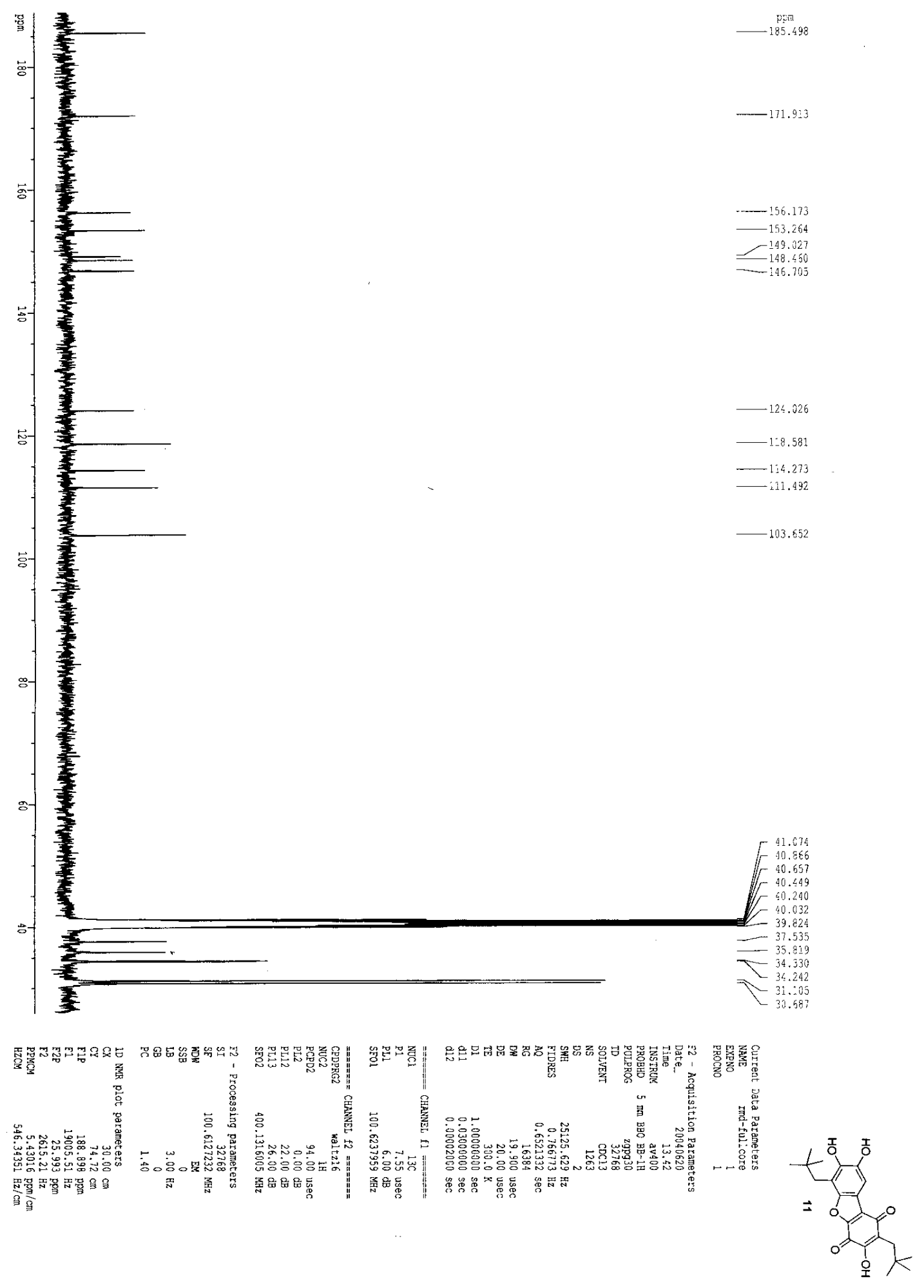




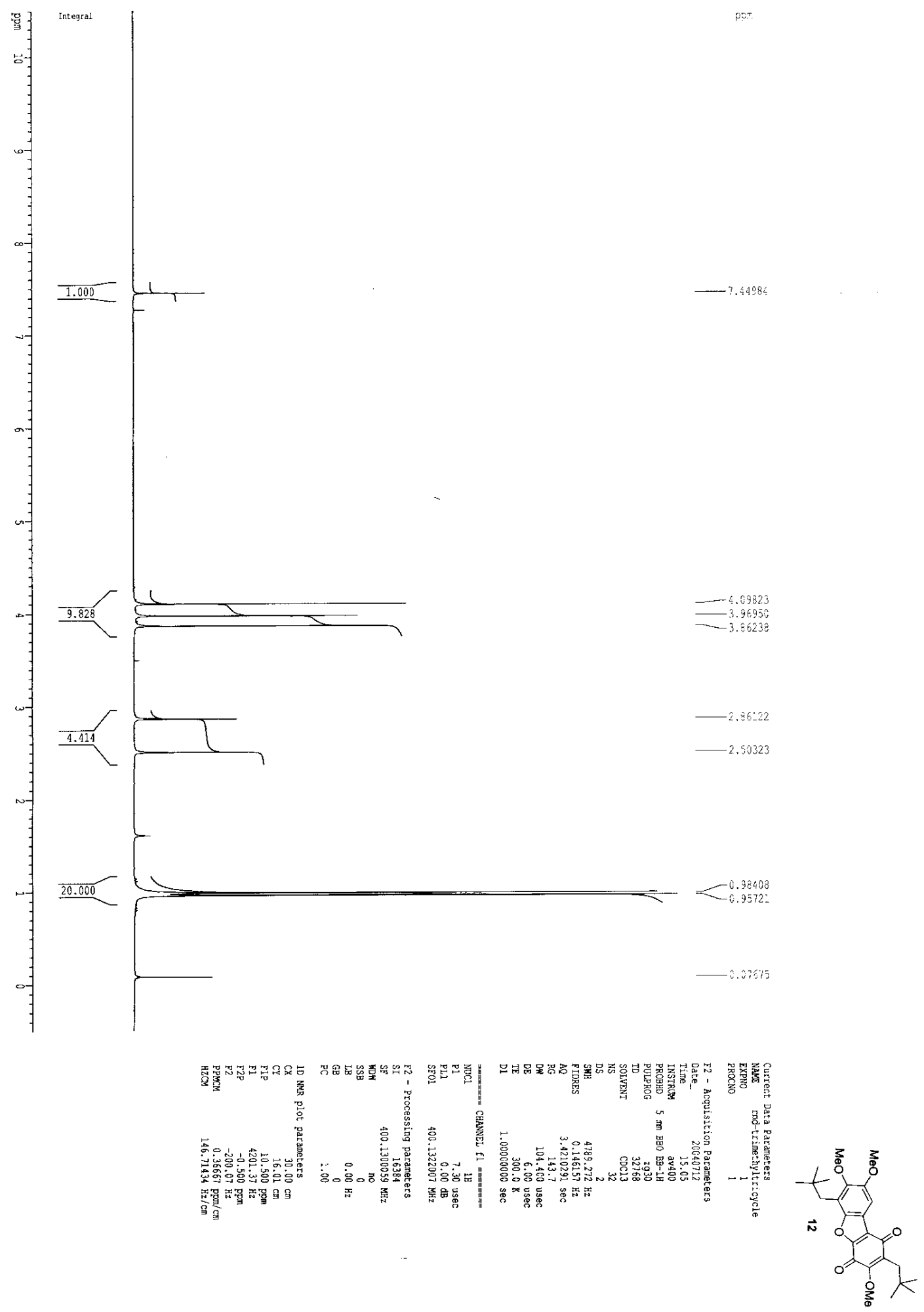




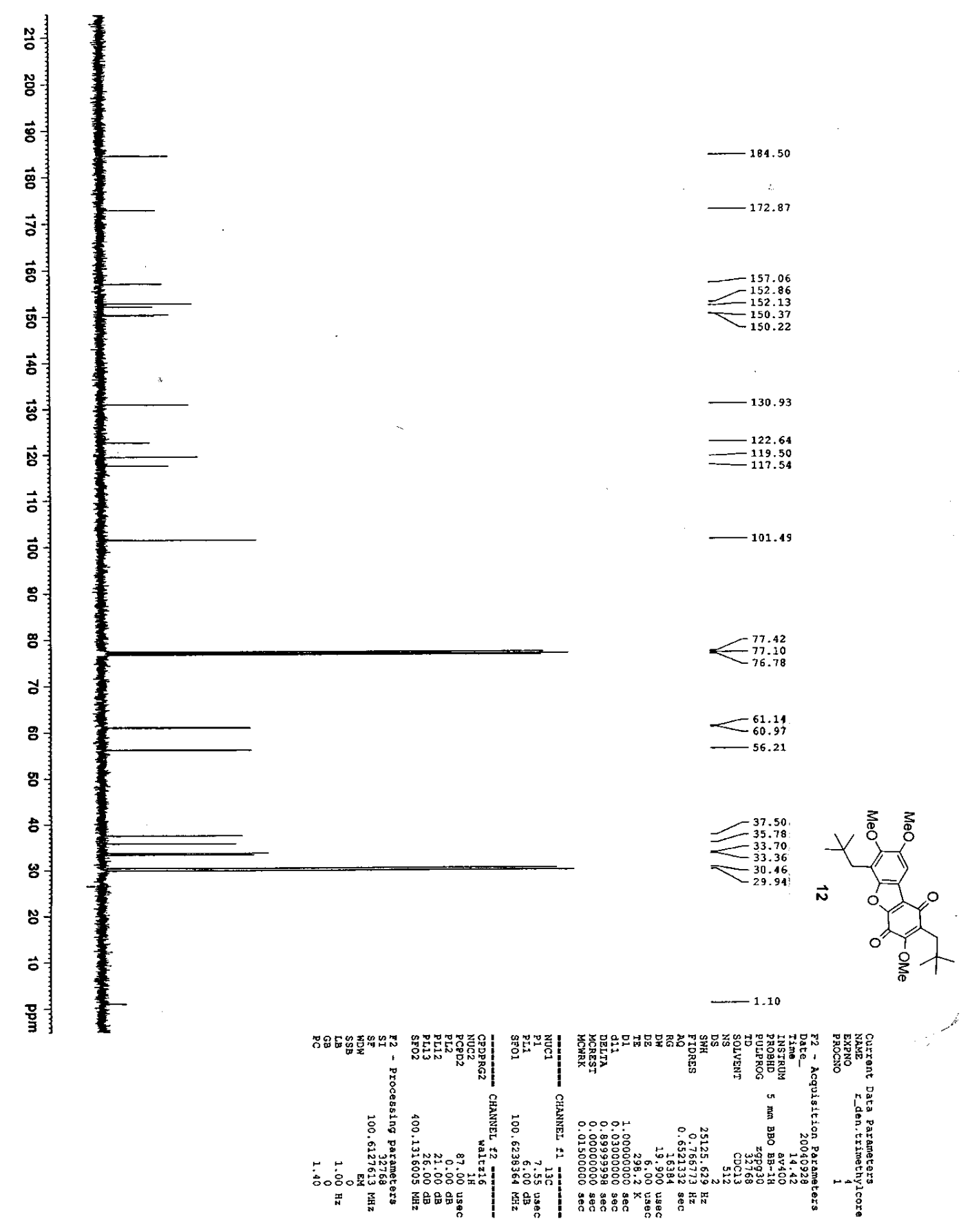



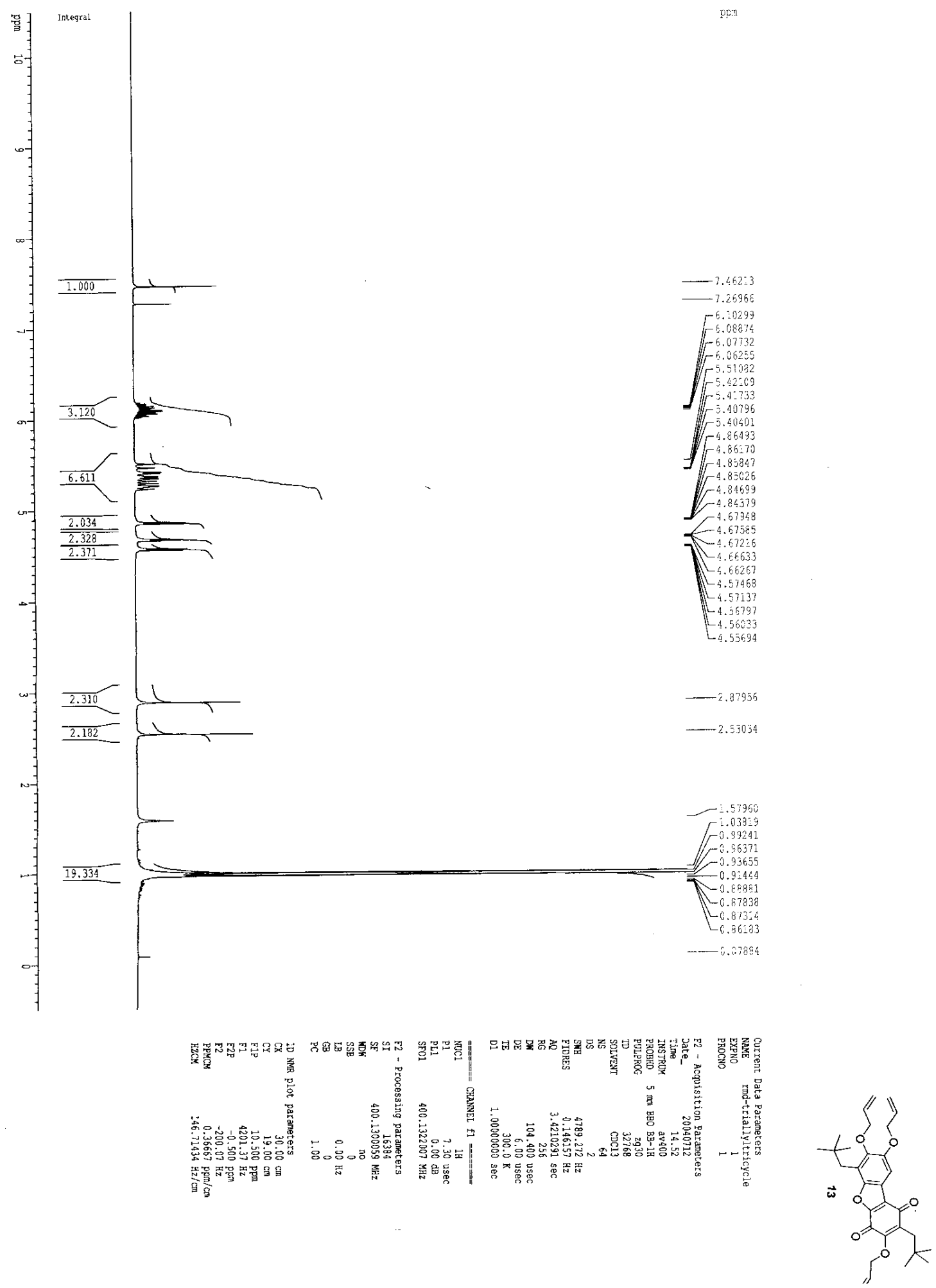


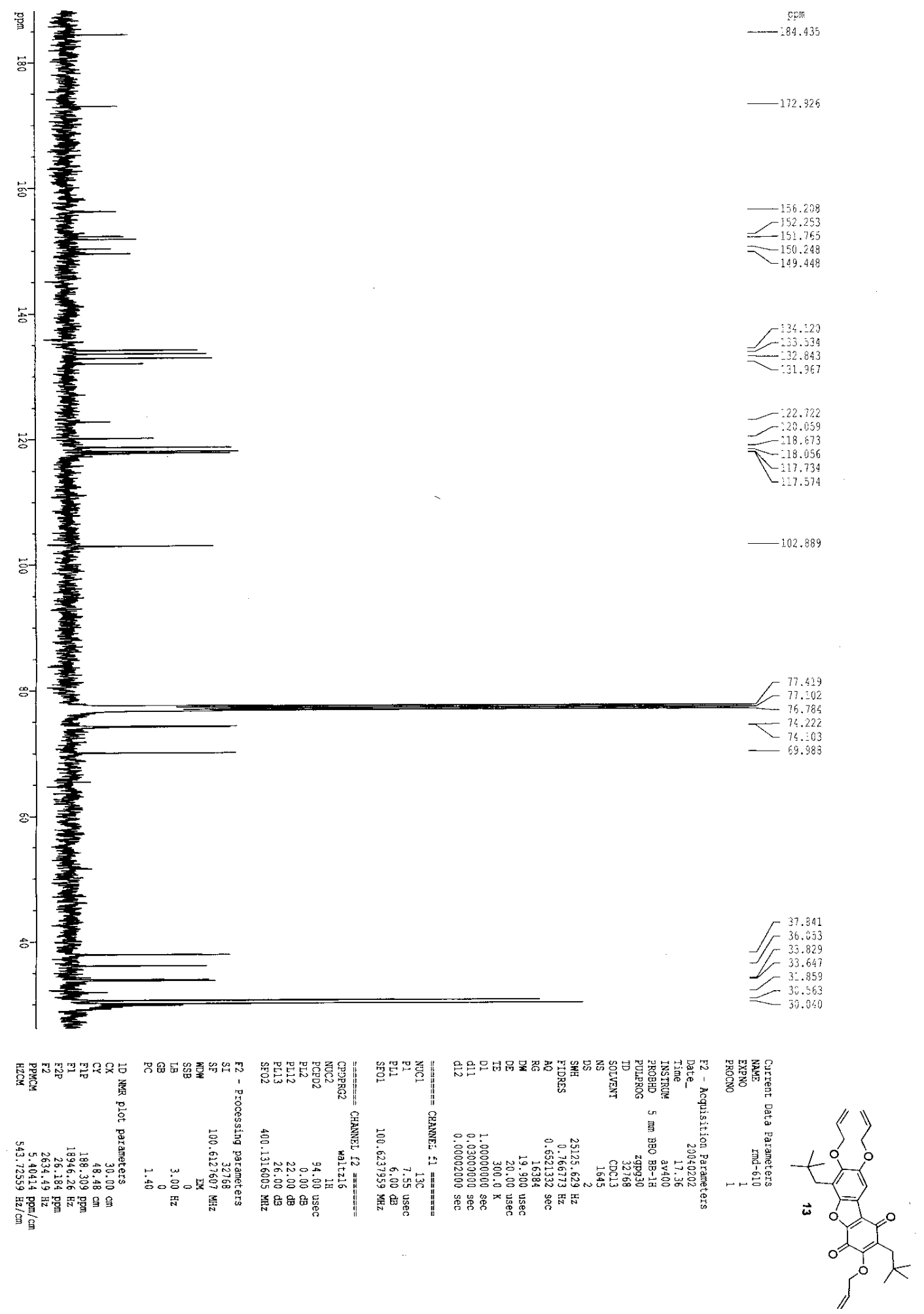



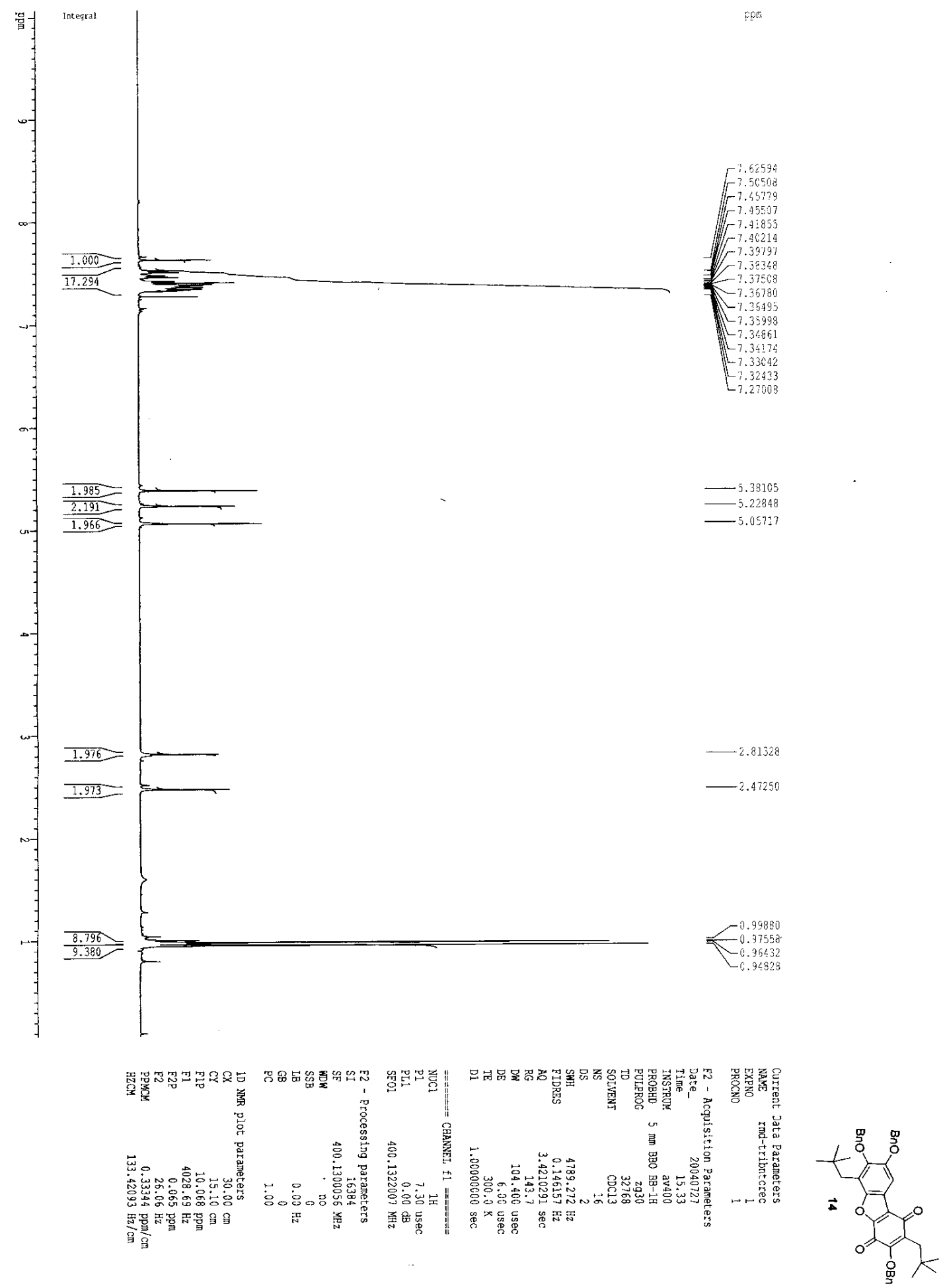

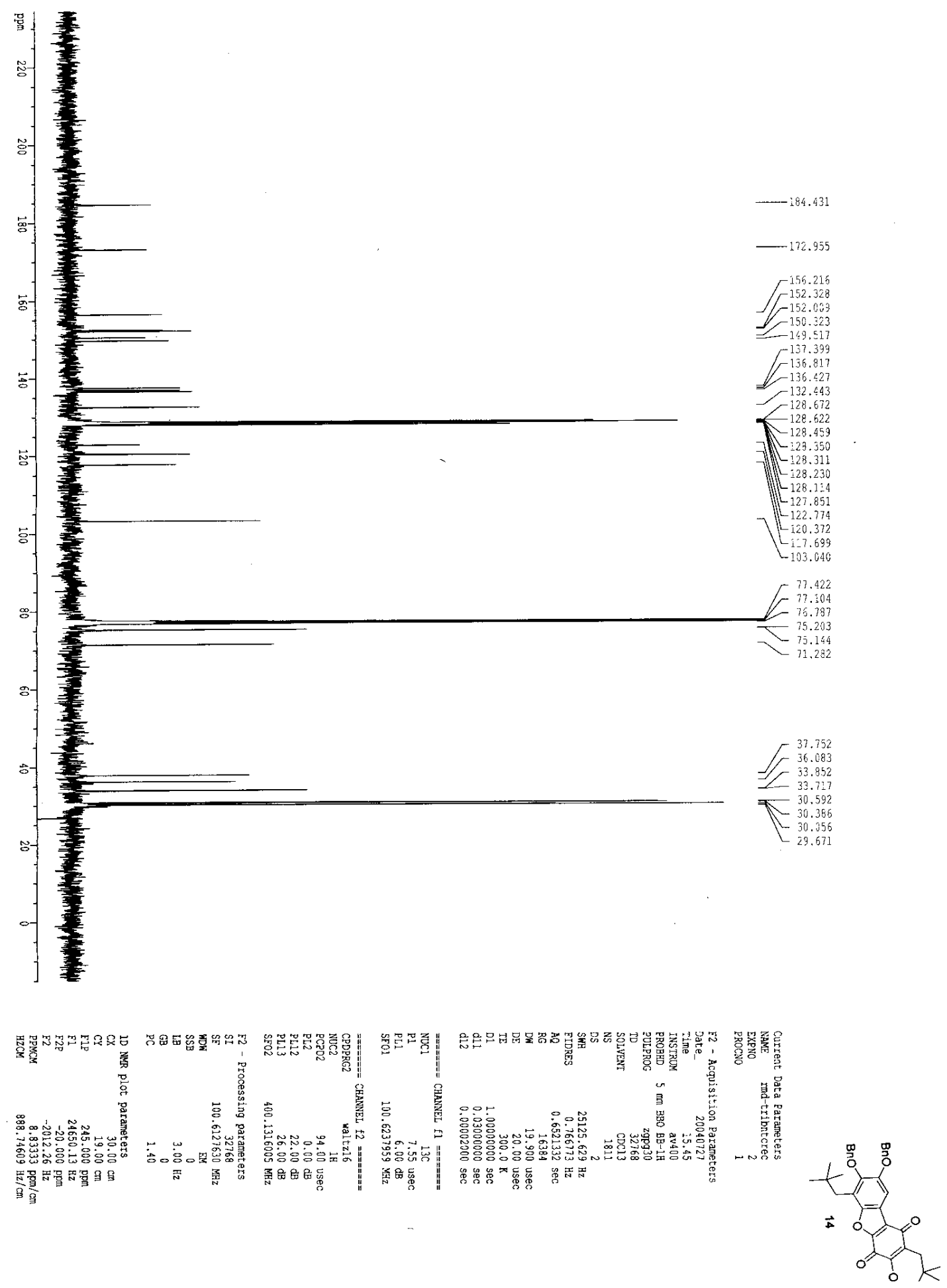

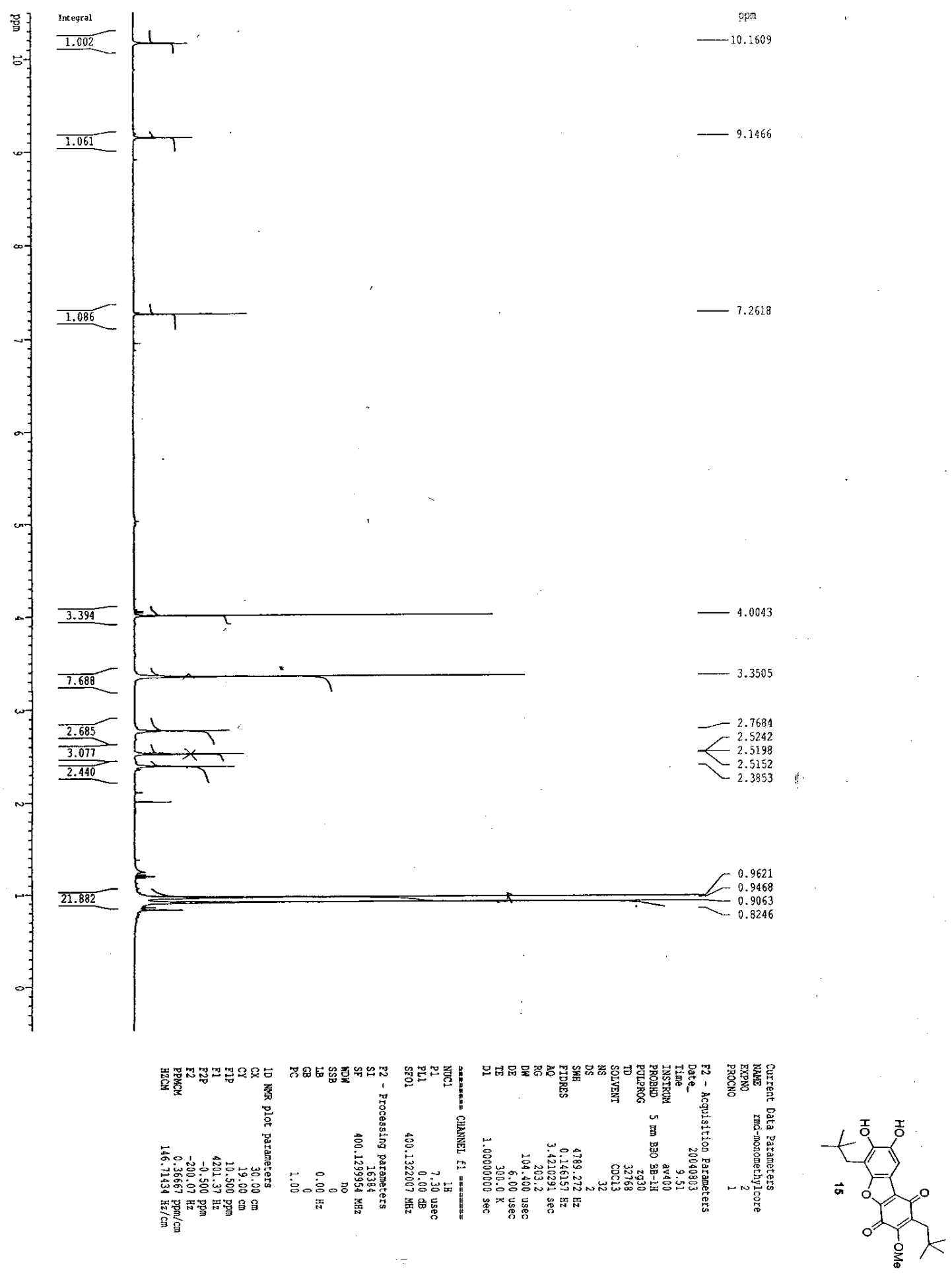


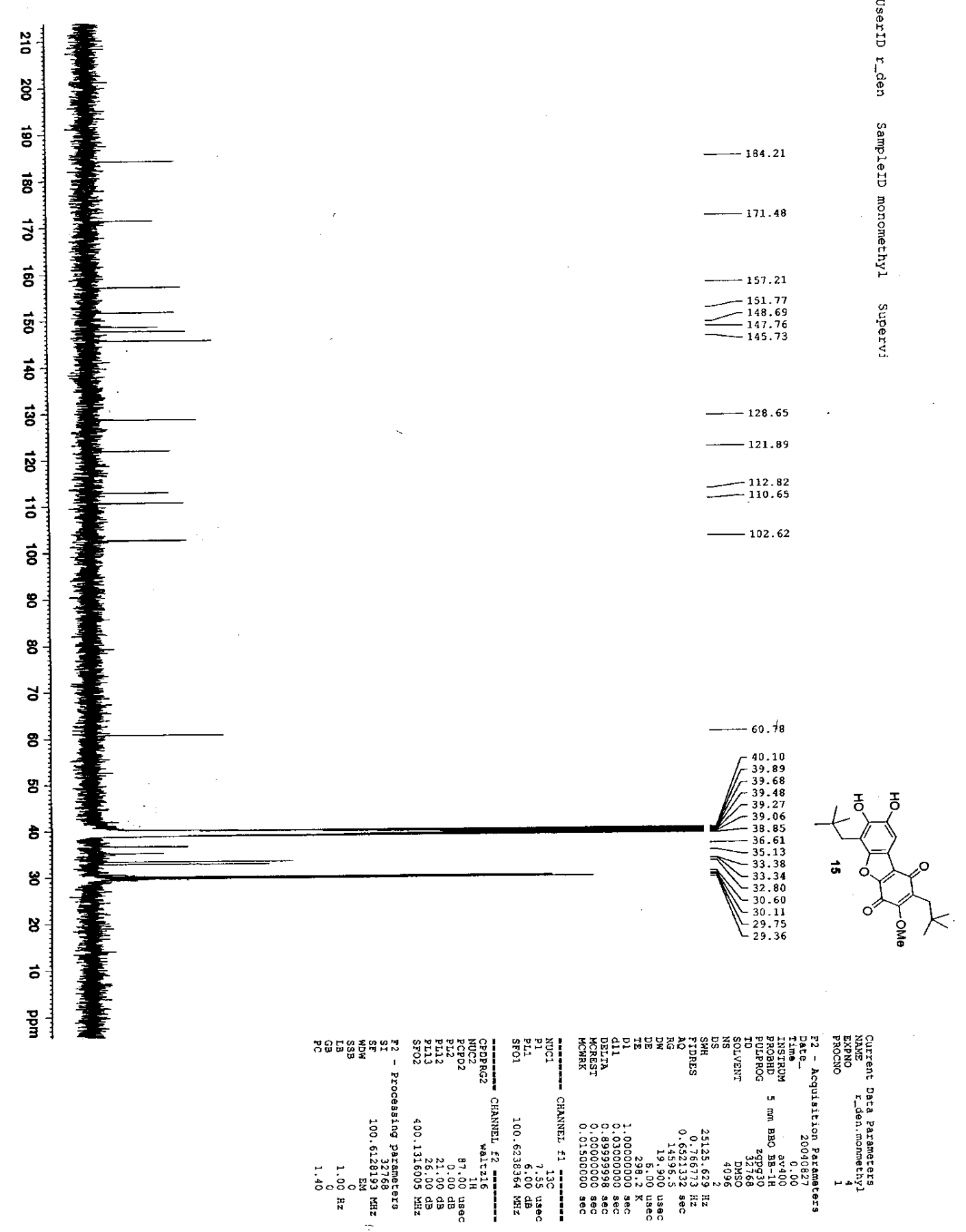




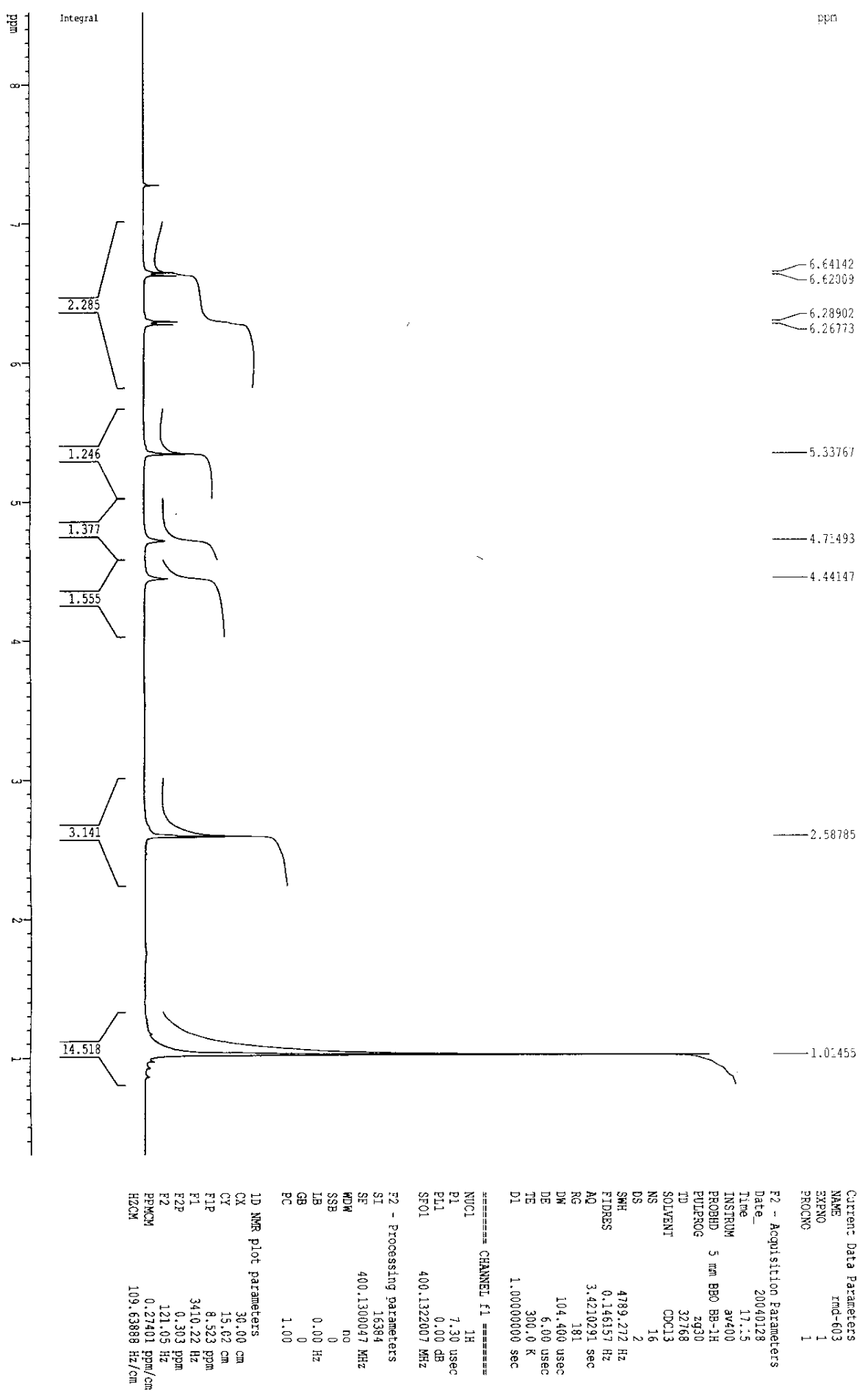




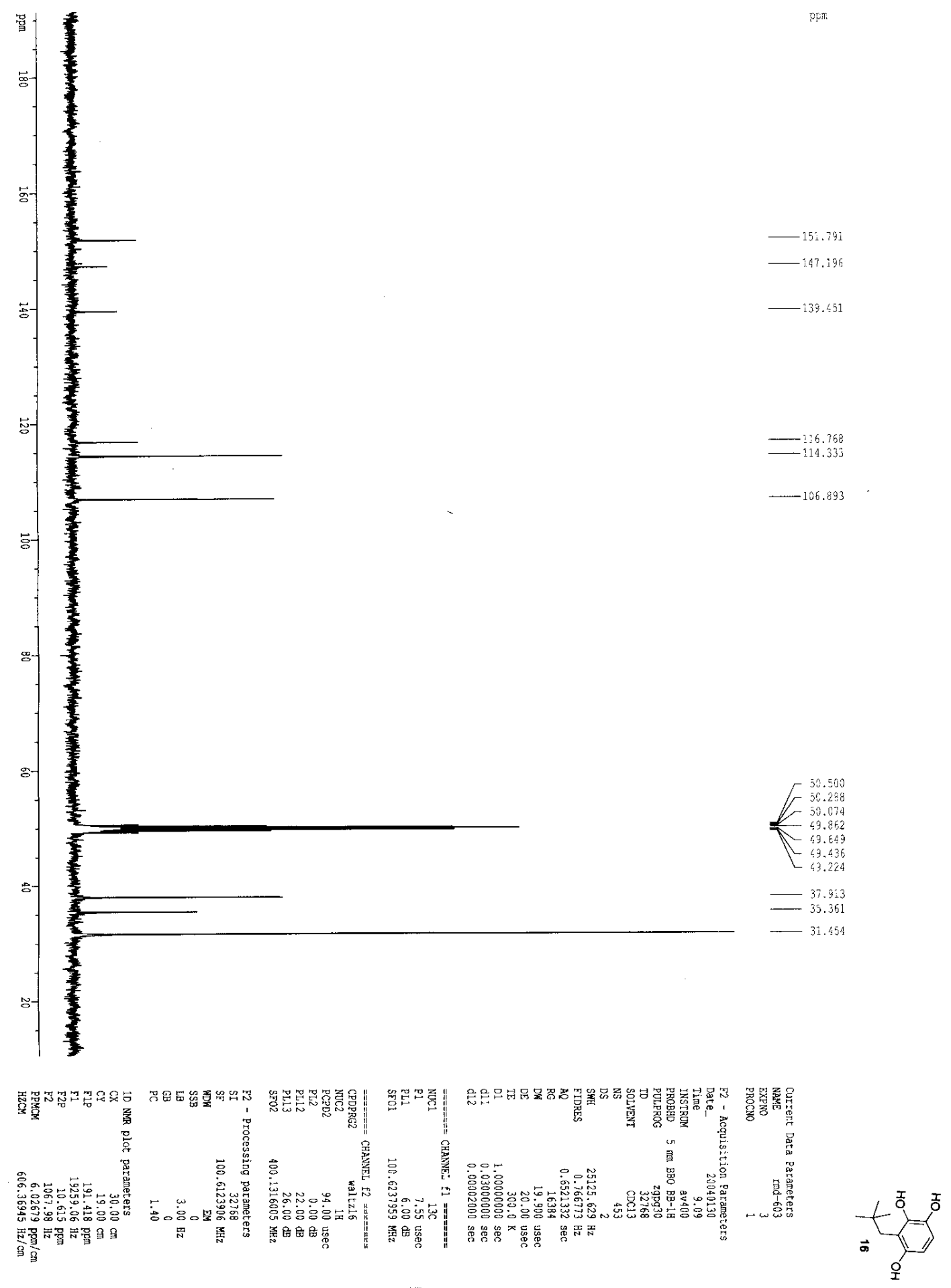

